\title{
The role of NPM, p14arf and MDM2 in precursors of bronchial squamous cell carcinoma
}

\author{
C. Mascaux*, F. Bex\#, B. Martin*, A. Burny ${ }^{\star}$, A. Haller ${ }^{+}$, M. Paesmans ${ }^{\S}$, \\ K. Willard-Gallo ${ }^{f}$, V. Ninane ${ }^{* *}$ and J-P. Sculier*
}

ABSTRACT: Murine double minute clone 2 (MDM2), p14 alternate reading frame (p14arf), and nucleophosmin (NPM) regulate p53 activity.

A total of 200 biopsies, including normal bronchial, pre-invasive and invasive tissues, were examined for changes in NPM, p14arf, MDM2 and p53 expression patterns by immunohistochemistry and immunofluorescence with confocal microscopy.

NPM and p14arf displayed a diffuse nuclear staining in most normal bronchial tissue. The fraction of biopsies displaying an increased MDM2 staining or a nucleolar relocalisation of NPM increased at mild and moderate dysplasia, respectively. Two different modifications occurred in p14arf expression, i.e. its loss or its nucleolar relocalisation, both increasing at severe dysplasia and both being associated with high MDM2 expression. In addition, the nucleolar relocalisation of p14arf was associated with that of NPM. Immunofluorescence staining indicated that NPM and p14arf either co-localised in the nucleoplasm or in the nucleoli, before and as a result of severe dysplasia, respectively. MDM2 was not detected in the nucleoli.

Thus, changes occur in murine double minute clone 2, p14 alternate reading frame and nucleophosmin level of expression and/or cellular distribution during early steps of lung carcinogenesis. Their relative localisation as determined by immunofluorescence, supports the hypothesis that p14 alternate reading frame nucleolar relocalisation impairs p14 alternate reading frame-murine double minute clone 2 complex formation and that nucleophosmin might sequester p14 alternate reading frame. The demonstration of this hypothesis requires further functional studies.

KEYWORDS: Carcinogenesis, lung cancer, murine double minute clone 2, nucleophosmin, p14 alternate reading frame

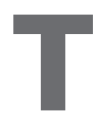
he tumour suppressor p53 is involved in the very early steps of lung carcinogenesis [1]. Its activity is regulated by several proteins, such as the human homologue of the murine double minute clone 2 (MDM2), p14 alternate reading frame (p14arf) and nucleophosmin (NPM; also called B23, numatrin or NO38). MDM2 plays an essential role in regulating p53 by blocking its transactivation domain and inducing its fast proteolysis; however, it also has oncogenic properties independent from p53 [2]. MDM2 is regulated by the tumour suppressor p14arf which binds to MDM2, preventing the p53-MDM2 interaction and thereby p53 degradation. It has been commonly accepted that p14arf sequesters MDM2 in the nucleoli [3, 4]. However, this model has now been challenged by studies showing that MDM2 is not relocalised from the nucleoplasm to the nucleoli during p14arf-induced growth arrest [5]. Recent studies suggest that NPM can sequester p14arf in nucleoli, preventing the formation of nucleoplasmic p14arf-MDM2 complexes [6]. NPM is indeed a protein involved in ribosome synthesis and protein shuttling, and it is now thought to play a role in carcinogenesis [7, 8], although the specific role of NPM in cancer is still under debate [8]. A potential oncogenic activity of this protein could be the nucleolar sequestration of p14arf [6].

At the early stages of lung carcinogenesis, the expression of p53 has been studied [1], but the expression of the proteins that regulate its activity has been poorly investigated. Indeed, MDM2 and p14arf have been widely studied in invasive lung cancer [9-25]. However, limited data suggest that MDM2 expression is modified in the early stages of
AFFILIATIONS

*Dept of Intensive Care and Thoracic Oncology,

${ }^{+}$Dept of Pathology,

${ }^{\S}$ Data Centre,

${ }^{f}$ Laboratory of Molecular

Immunology, Institut Jules Bordet,

ULB (Université Libre de Bruxelles),

\#Laboratory of Microbiology,

Institute for Microbiological Research J-M Wiame,

-Laboratory of Molecular and

Cellular Biology, Faculté Universitaire des Sciences Agronomiques de

Gembloux (FUSAGx), and

**Dept of Pneumology, CHU Saint-

Pierre, ULB (Université Libre de

Bruxelles), Brussels, Belgium.

CORRESPONDENCE

C. Mascaux, Institut Jules Bordet,

Rue Héger-Bordet 1, B-1000

Brussels, Belgium.

Fax: 3225343756

E-mail: celine.mascaux@bordet.be

Received:

January 172008

Accepted after revision:

April 302008

SUPPORT STATEMENT

This study was supported by a grant from the Fonds de la Recherche

Scientifique Medicale (FRSM)

Brussels, Belgium, a grant from the Télévie Fonds National de la Recherche Scientifique (FNRS), Belgium, and a grant from the ASBL "Les Amis de l'Institut Jules Bordet" Brussels, Belgium. C. Mascaux is a research fellow for the FNRS and

K. Willard-Gallo is a scientific collaborator of the FNRS-Télévie.

STATEMENT OF INTEREST

None declared.

European Respiratory Journal

Print ISSN 0903-1936

Online ISSN 1399-3003 
TABLE 1 Distribution of aberrant murine double minute clone 2 (MDM2), nucleophosmin (NPM) and p14 alternate reading frame (arf) expression according to histological classification

\begin{tabular}{|c|c|c|c|c|c|}
\hline \multirow[t]{2}{*}{ Histology } & \multirow[t]{2}{*}{ High MDM2 } & \multirow{2}{*}{$\begin{array}{l}\text { NPM nucleolar } \\
\text { relocalised }\end{array}$} & \multicolumn{3}{|c|}{ p14arf } \\
\hline & & & Negative & Nucleolar relocalised & Aberrant expression \\
\hline Normal & 5/26 (19) & $0 / 15(0)$ & 4/16 (25) & $0 / 16(0)$ & 4/16 (25) \\
\hline Hyperplasia & 8/36 (22) & 2/22 (9) & 4/26 (15) & $1 / 26(4)$ & 5/26 (19) \\
\hline Metaplasia & 9/32 (28) & 1/22 (5) & 4/24 (17) & $1 / 24(4)$ & $5 / 24(21)$ \\
\hline Severe dysplasia & 15/19 (76) & $4 / 18(22)$ & $9 / 18(50)^{\star \star \star *}$ & $4 / 18(22)^{\star \star \star \star 丿 ~}$ & $13 / 18(72)^{\star \star \star \cdot}$ \\
\hline In situ carcinoma & $13 / 16(81)$ & 2/16 (13) & $9 / 16(56)$ & 2/16 (18) & $11 / 16(74)$ \\
\hline Invasive carcinoma & $9 / 12(75)$ & $6 / 10(60)$ & $5 / 10(50)$ & 2/10 (20) & $7 / 10(70)$ \\
\hline Overall & $89 / 179(50)$ & 20/136 (15) & $42 / 144(29)$ & $13 / 144(9)$ & $55 / 144(38)$ \\
\hline
\end{tabular}

Data are expressed as number of biopsies with aberrant protein expressed/total number of assessed biopsies (\%). \#: total of negative and nucleolar localised groups

$\because$ stage at which protein expression changed significantly. ${ }^{+}: p=0.001 ;{ }^{* *}: p<0.001 ; p$-values obtained by Chi-squared test for a linear trend.

carcinogenesis [14], while p14arf has not been assessed in bronchial pre-invasive tissues. To the current authors' knowledge, NPM expression has not been studied in lung cancer, either at invasive or early stages. The purpose of the present study was to assess whether MDM2, p14arf and NPM are expressed, and to determine whether their expression level and/or distributions are modified, during early squamous cell carcinogenesis. In addition, the present study analysed if any relationship exists between the expression levels and/or distribution of p53, MDM2, p14arf and NPM in bronchial pre-neoplasia.

\section{MATERIALS AND METHODS}

\section{Study population}

Eligible patients had a minimum smoking exposure of 30 packyrs and/or a history of lung or head and neck cancer. Fluorescence bronchoscopy was performed under local anaesthesia and all areas that appeared as abnormal were biopsied [26]. Only the most severe lesion found in an individual patient was examined and every biopsy analysed was from a different patient. In total, 200 consecutively eligible patients were included in the study between 1996 and 2002. The slides used to assess MDM2, p14arf and NPM were successively cut from the paraffin block; however, some blocks contained insufficient tissue for assessment of all three proteins. The numbers of studied biopsies for MDM2, p14arf and NPM expression were 179, 144 and 136, respectively. All stained biopsies were classified using the 1999 histological World Health Organization/International Association for the Study of Lung Cancer [27] classification of pre-invasive and invasive squamous lesions of the bronchus into the following groups: 1) microscopically normal bronchial epithelium from smokers or nonsmokers (normal); 2) hyperplasia; 3) metaplasia; 4) mild dysplasia (MiD); 5) moderate dysplasia $(\mathrm{MoD})$; 6) severe dysplasia (SD); 7) carcinoma in situ; and 8) invasive squamous cell carcinoma (SQCC; table 1) [27].

\section{Immunohistochemistry for MDM2, p14arf and NPM}

Biopsies were routinely fixed in $10 \%$ neutral buffered formalin and embedded in paraffin. Tissue slides $(4 \mu \mathrm{m})$ were deposited on SuperFrost Plus Slides (Menzel-Gläser, Braunschweing, Germany). Immunohistochemistry (IHC) was performed using a standard avidin-biotin-peroxidase complex [28, 29]. For MDM2 staining, antigen retrieval was accomplished by microwave heating for $15 \mathrm{~min}(650 \mathrm{~W})$ in $0.01 \mathrm{M}$ citrate buffer, pH 6 and, for p14 and NPM, by immersion in a basic solution (Tris-EDTA buffer, $\mathrm{pH}$ 9) for $30 \mathrm{~min}$ in a boiling water bath. All of the following steps were performed automatically at $37^{\circ} \mathrm{C}$ using the NexES system (Ventana Medical Systems, Tucson, AZ, USA). The slides were incubated with the antiNPM antibodies (mouse monoclonal immunoglobulin (Ig)G1; B23 (0412); sc-47725 dilution 1/150; Santa Cruz Biotechnology, Santa Cruz, CA, USA) for $30 \mathrm{~min}$ at $37^{\circ} \mathrm{C}$ or $60 \mathrm{~min}$ at $37^{\circ} \mathrm{C}$ with the anti-MDM2 antibody (mouse monoclonal IgM; NCLMDM2 dilution 1/50; Novocastra, Newcastle upon Tyne, UK) or overnight at $4{ }^{\circ} \mathrm{C}$ with the anti-p14arf antibody (rabbit polyclonal IgG; ab3642 dilution 1/100, Abcam; Novus Biological, Littleton, CO, USA).

Negative controls included omitting the primary antibody and substituting it with a normal mouse IgM for the MDM2 antibody, a normal rabbit IgG for p14arf and a normal mouse IgG1 for NPM. MDM2 positive osteosarcoma, p14arf and NPM positive cervix carcinoma were used as positive controls.

Three independent investigators assessed IHC stainings. Individual observations were compared and discordant interpretations were resolved by a collective review of the slides in question using a multi-head microscope. All analyses were performed blind to patient background information. For the three proteins, only nuclear staining was assessed, since cytoplasmic staining was considered as background [21, 24, 30].

\section{Immunofluorescence for p53, MDM2, p14arf and NPM}

After blocking for $30 \mathrm{~min}$ with PBS containing $0.5 \%$ gelatine (Bio-Rad, Hercules, CA, USA) and $0.25 \%$ bovine serum albumin (Gibco, Invitrogen, Carlsbad, CA, USA), the slides were incubated overnight at $4{ }^{\circ} \mathrm{C}$ in humid chambers with the primary antibodies (anti-p53 (IgG2bк; clone DO-7, MS186 R7; 
DakoCytomation, Copenhagen, Denmark), dilution 1/50; anti-MDM2, dilution 1/50; anti-p14arf, dilution 1/25; and anti-NPM, dilution 1/100). After washing with PBS containing $0.2 \%$ gelatine, the slides were incubated with the secondary antibodies in humid chambers for $60 \mathrm{~min}$ at room temperature. Goat anti-mouse IgG conjugated to Alexa Fluor 633 (1/100; Molecular Probes, Eugene, OR, USA) was used to recognise the anti-p53 antibody; goat anti-mouse IgM conjugated to fluorescein isothiocyanate (1/50; Jackson Immunoresearch, West Grove, PA, USA) for anti-MDM2 antibody; goat anti-rabbit IgG conjugated to Alexa Fluor 546 (1/100; Molecular Probes) for anti-p14arf antibody; and goat anti-mouse IgG1 coupled to biotin (1/100; Southern Biotechnology Associates, Birmingham, AL, USA) for anti-NPM antibody. When the secondary antibody was the biotinylated goat anti-mouse IgG1, the slides were incubated for 20 min with Alexa Fluor 633 conjugated strepatvidin (dilution 1/1000; Molecular Probes). The samples were washed again with PBS containing $0.2 \%$ gelatine and mounted in DABCO (1, 4-diazabicyclo[2.2.2] octane)-based medium (MP Biomedicals, Irvine, CA, USA). Two negative controls demonstrated the desired specificity of the fluorescent reagents by: 1) omission of the primary antibody; and 2) staining negative slides identified using IHC staining for each individual protein. External positive controls were those used in IHC.

The slides were analysed with a laser scanning confocal microscope (LSM 510; Carl Zeiss Microscopy, Iena, Germany) using a $63 \times$ objective and light source wavelengths of 488 , 546 and $633 \mathrm{~nm}$. The previously described negative controls were used as references to determine the zero intensity level of staining. The slides were successively analysed at each light source wavelengths to identify the presence of each individual protein. The protein's cellular localisation was assessed by determining the corresponding differential interference contrast (DIC). The projection of the three confocal images with the three light source wavelengths was referred to as "overlay images". The relative intracellular localisation of the four proteins was assessed by analysing the fluorescence intensity profiles for each staining along lines drawn across representative nuclei on the zoom of the overlay images.

\section{Statistical assessment}

The correlation between two continuous variables was assessed using the Spearman rank correlation coefficient. Mann-Whitney and Kruskal-Wallis tests were performed to compare the distribution of continuous variables based on binary or categorical values (more than two categories), respectively. Comparison between the distributions of two dichotomic variables was performed using Fisher bilateral exact tests. Comparisons between the distributions of a categorical (more than two categories) and dichotomic or two categorical variables, were assessed using Chi-squared tests for heterogeneity or for a trend in case of ordering of the level of the categorical variable, respectively.

The current authors are aware that performing multiple tests increases the probability of false-positives. For the present study's exploratory purpose, the threshold level for statistical significance was reduced from 0.05 to 0.01 .

\section{RESULTS}

\section{Evaluation of MDM2, p14arf and NPM expression using IHC}

MDM2 expression

The distribution of MDM2 staining was scored according to EYMIN et al. [21] (fig. 1). Based on this scoring system, 89 out of $179(50 \%)$ biopsies overexpressed MDM2 (referred to in the text as high MDM2). The distribution of MDM2 expression in relation to histological stage is shown in table 1. A significant increase in the number of high MDM2-expressing biopsies occurred at MiD and remained stable from MiD to SQCC. Two groups were thus observed: group 1 and group 2 with $23 \%$ and $79 \%$ high MDM2, respectively $(\mathrm{p}<0.001)$.

\section{p14arf expression}

p14arf expression was considered negative when $<10 \%$ of the cells were stained $[15,19,20]$. Among the positive slides, two patterns were observed: either the nuclei were totally stained or the staining was localised in small round nuclear structures,
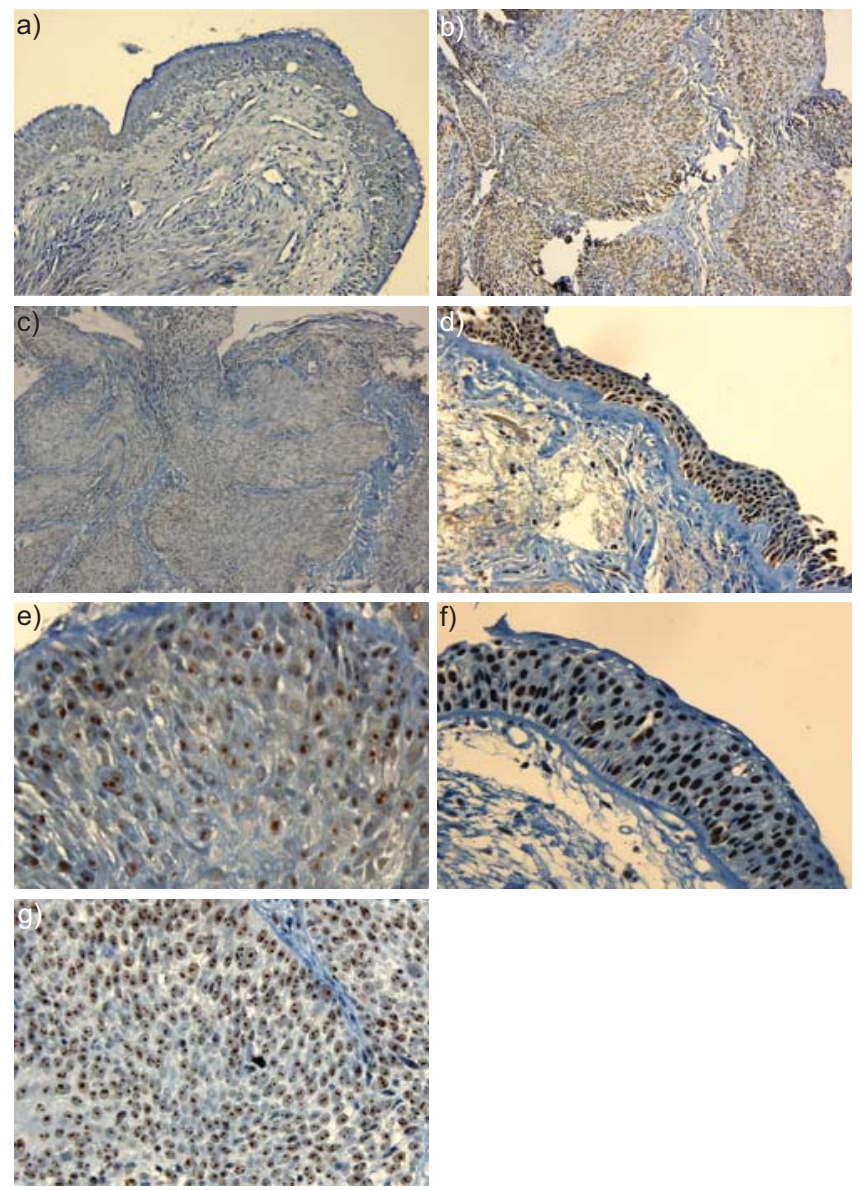

FIGURE 1. Immunohistochemical stainings showing the different patterns of expression for the three proteins. a) Low murine double minute clone 2 (MDM2) expression (score 1 according to EYMIN et al. [21]) in a hyperplasia; b) in situ carcinoma highly expressing MDM2 (score 3 according to EYMIN et al. [21]); c) in situ carcinoma (same biopsy as b), which is negative for p14 alternate reading frame (p14arf); d) metaplasia showing diffuse nuclear p14arf expression; e) moderate dysplasia showing a nucleolar concentration of p14arf; f) moderate dysplasia showing diffuse nuclear nucleophosmin (NPM) expression; and g) in situ carcinoma showing a nucleolar relocalisation of NPM. 
identified by immunofluorescence (IF) as nucleoli. The slides showing $\geqslant 10 \%$ of cells with p14arf relocalised from the nucleoplasm to the nucleoli were designated nucleolar relocalised, while the remaining positive slides $(\geqslant 10 \%$ of cells stained but $<10 \%$ of cells with relocalised p14arf in the nucleoli) were designated diffuse nuclear. The different patterns of expression are shown in figure 1 . Among the 144 biopsies assessed for p14arf expression, 42 (29\%) biopsies had lost p14arf expression based on a cut-off at 10\% (p14arfnegative). In the group of 102 p14arf-positive biopsies, 13 (9\% of all biopsies) displayed p14arf nucleolar redistribution and 89 ( $62 \%$ of all biopsies) had diffuse nuclear p14arf staining. The distribution of p14arf expression in three subgroups (negative, relocalised to nucleoli, and diffuse nuclear) relative to the histopathological classification is shown in table 1 . The proportion of p14arf-negative biopsies was lower in biopsies with a grade inferior to SD (19 versus $52 \% ; \mathrm{p}<0.001$ ). The percentage of biopsies with redistributed p14arf nucleolar staining also increased from SD (38 versus $6 \% ; \mathrm{p}<0.001)$. The proportion of aberrant p14arf expression, including the loss of expression or nucleolar redistribution, also increased at the stage of SD (70 versus $24 \%$; $<<0.001$ ).

\begin{tabular}{|c|c|c|c|c|}
\hline TABLE 2 & $\begin{array}{l}\text { Distribution of } \\
\text { (p14arf) expressi } \\
\text { minute clone } 2\end{array}$ & $\begin{array}{l}\text { alternate } \\
\text { accord } \\
\text { M2) ex }\end{array}$ & $\begin{array}{l}\text { eading frar } \\
\text { g to murine } \\
\text { ession }\end{array}$ & double \\
\hline \multirow[t]{2}{*}{ MDM2 } & & \multicolumn{3}{|c|}{ p14arf } \\
\hline & & $\begin{array}{l}\text { Diffuse } \\
\text { nuclear }\end{array}$ & $\begin{array}{l}\text { Nucleolar } \\
\text { relocalised }\end{array}$ & Negative \\
\hline \multicolumn{5}{|l|}{ All biopsies } \\
\hline High & & 31 & $11^{\#}$ & $30^{\#}$ \\
\hline Low & & $56^{\#}$ & 2 & 10 \\
\hline Total & & 87 & 13 & 40 \\
\hline \multicolumn{5}{|c|}{ Stages preceding severe dysplasia } \\
\hline High & & 22 & 4 & 12 \\
\hline Low & & $52^{\circ}$ & 1 & 6 \\
\hline Total & & 74 & 5 & 18 \\
\hline \multicolumn{5}{|c|}{ Stages from severe dysplasia } \\
\hline High & & 9 & 7 & $18^{\bullet}$ \\
\hline Low & & 4 & 1 & 4 \\
\hline Total & & 13 & 8 & 22 \\
\hline \multicolumn{5}{|c|}{$\begin{array}{l}\text { \#: the largest group for each pattern of p14arf expression. }{ }^{\circ} \text { largest group of } \\
\text { that table. In all biopsies, a statistically significant }(\mathrm{p}<0.001) \text { association was } \\
\text { found between the distributions of p14arf and MDM2 patterns of expression. } \\
\text { Indeed, the majority of biopsies with loss ( } 30 \text { out of } 40: 75 \%) \text { or a nucleolar } \\
\text { relocalisation ( } 11 \text { out of } 13: 85 \% \text { ) of p14arf expression, also displayed MDM2 } \\
\text { high expression. On the opposite, } 64 \% \text { ( } 56 \text { out of } 87 \text { ) of biopsies with p14arf } \\
\text { diffuse nuclear staining did not aberrantly express MDM2. The association } \\
\text { between p14arf and MDM2 is shown preceding and from the stages of severe } \\
\text { dysplasia at which p14arf aberration increases statistically. In stages preceding } \\
\text { severe dysplasia, the largest group has diffuse nuclear p14arf expression and } \\
\text { low MDM2 expression. In stages from severe dysplasia, the largest group loses } \\
\text { p14arf expression and shows high MDM2 expression. The statistical } \\
\text { significance of these data could not be calculated due to the small number } \\
\text { of samples. }\end{array}$} \\
\hline
\end{tabular}

\section{NPM expression}

Two different patterns of staining were observed for NPM in bronchial epithelial cells: either diffuse nuclear staining or concentrated only in the nucleoli with no staining detected in the nucleoplasm. The first pattern is referred to as diffuse nuclear staining and the second as nucleolar relocalised staining (fig. 1) [30]. Among the 136 biopsies assessed for NPM expression, $20(15 \%)$ biopsies had aberrant NPM expression with NPM being totally relocalised to the nucleoli. The abnormal distribution of NPM in relation to histological stage is shown in table 1 . There was a statistically significant increase in NPM relocalisation to the nucleoli in MoD. Two different subgroups of NPM expression were thus identified: group 1 and group 2 with 5 and $26 \%$ of the biopsies showing NPM relocalised to the nucleoli, respectively $(p=0.001)$.

\section{Correlations of protein expression}

The expression of p14arf and MDM2 was assessed in 140 biopsies. An association (table 2) between the pattern of p14arf (negative, nucleolar relocalised, diffuse nuclear) and MDM2

\begin{tabular}{llll} 
TABLE 3 & $\begin{array}{l}\text { Distribution of nucleophosmin (NPM) expression } \\
\text { based on p14 alternate reading frame (p14arf) } \\
\text { expression }\end{array}$ \\
\cline { 2 - 4 } NPM & \multicolumn{2}{c}{$\begin{array}{c}\text { p14arf } \\
\text { Diffuse } \\
\text { nuclear }\end{array}$} & $\begin{array}{c}\text { Nucleolar } \\
\text { relocalised }\end{array}$ \\
\hline
\end{tabular}

\section{All biopsies}

Diffuse nuclear

Nucleolar relocalised

Total

$\begin{array}{ccc}78^{\#} & 3 & 34^{\#} \\ 5 & 10^{\#} & 5 \\ 83 & 13 & 39 \\ & & \\ 67^{*} & 2 & 14 \\ 3 & 3 & 2 \\ 70 & 5 & 16 \\ & & \\ 11 & 1 & 20^{\bullet} \\ 2 & 7^{\bullet} & 3 \\ 13 & 8 & 23\end{array}$

Stages preceding severe dysplasia

Diffuse nuclear

Nucleolar relocalised

Total

Stages from severe dysplasia

Diffuse nuclear

Nucleolar relocalised

Total

23

\#: largest group for each pattern of p14arf expression. ":largest group of that table. Nucleolar relocalisations of NPM and p14arf were consistently related (Chi-squared, $\mathrm{p}<0.001$ ), $77 \%$ (10 out of 13) of biopsies with nucleolar p14arf also had NPM relocalised in the nucleoli. Diffuse nuclear NPM staining was observed in $87 \%$ (34 out of 39 ) of p14arf biopsies and $94 \%$ (78 out of 83 ) of biopsies with diffuse nuclear p14arf staining. Results from biopsies preceding and from severe dysplasia illustrate the association between p14arf and NPM before and from the stage of severe dysplasia onwards at which p14arf aberration statistically increases. In biopsies preceding severe dysplasia, the largest group shows diffuse nuclear p14arf and diffuse nuclear NPM expression. In stages from severe dysplasia, the largest group loses p14arf expression and shows nuclear NPM expression. In addition, the number of biopsies with nucleolar relocalised NPM increases and the majority (seven out of eight) also have a nucleolar relocalisation of p14arf. The statistical significance of these data could not be calculated due to the small number of samples. 
expression (Chi-squared; $\mathrm{p}<0.001$ ) was found. The relocalisation of p14arf to the nucleoli or the loss of its expression were both associated with high MDM2 expression.

A total of 135 biopsies were assessed for the expression of the three proteins. Aberrant NPM expression was not associated with aberrant MDM2 expression $(\mathrm{p}=0.144)$. Nucleolar relocalisation of NPM and p14arf were consistently associated (Chisquared, $\mathrm{p}<0.001$; table 3 ).

\section{Relative intracellular distributions assessed by IF staining and confocal microscopy}

Sections from selected samples representing the different IHC patterns detected for these three markers were analysed using triple IF staining with antibodies to either p14arf, MDM2 and p53 (13 biopsies: two hyperplasia, three metaplasia, four MoD, two SD and two SQCC, with three representative samples shown in figures 2, 3 and 4) or p14arf, MDM2 and NPM (11 biopsies: two hyperplasia, two metaplasia, one MiD, four MoD, two SQCC, with two representative samples shown in figures 5 and 6).

First, the intracellular distribution of these proteins was assessed. IF confirmed the majority of the results obtained by IHC, and provided additional information about the intracellular localisation of proteins like MDM2 and p53, which were excluded from the nucleoli (not seen by IHC) and were distributed in the nucleoplasm in all biopsies analysed for MDM2 (figs 2-6) and in biopsies in which p53 was expressed (figs 2 and 3). However, p53 was undetectable in six of 13 samples (fig. 4). The three and the two different patterns observed by IHC for p14arf (figs 2-6) and for NPM (figs 5 and 6), respectively, were confirmed by IF. Moreover, while both p14arf and NPM displayed a diffuse distribution in the whole nuclei on IHC, IF showed that p14arf was exclusively localised in the nucleoplasm (fig. 5) in these cases. IF also identified the nuclear loci in which p14arf and NPM co-localised as being the nucleoli.

Secondly, the relative intracellular localisations of these proteins were determined. Fluorescence intensity profiles showed that MDM2 (green) and p53 (blue) co-localised in the nucleoplasm (figs 2 and 3). The localisation of p14arf relative to nucleoplasmic and/or nucleolar NPM was also assessed. When NPM was present in both the nucleoli and the nucleoplasm, it co-localised exclusively with p14arf in the nucleoplasm (fig. 5). When NPM was present only in the nucleoli, it co-localised with p14arf in the nucleoli (fig. 6). MDM2 was consistently detected in the nucleoplasm, excluding the nucleoli, and this distribution was independent from the absence of p14arf (fig. 2) or from its distribution either in the nucleoplasm (fig. 5) or in the nucleoli (figs 3, 4 and 6).

\section{DISCUSSION}

The present data, based on a large series of biopsies, indicated that aberrant expression of MDM2, NPM and p14arf appeared at different stages during bronchial squamous cell carcinogenesis: MiD, MoD and SD, respectively. Two different types of aberrations were observed in p14arf expression, loss of the protein or its relocalisation to nucleoli. Both types of aberrances in p14arf expression, loss or relocalisation, statistically increased at SD and were associated with a high level of MDM2 expression. p14arf nucleolar relocalisation was correlated to that of NPM. NPM and p14arf co-localised either in the nucleoplasm or the nucleoli. Interestingly, MDM2 was not found relocalised to the nucleoli.
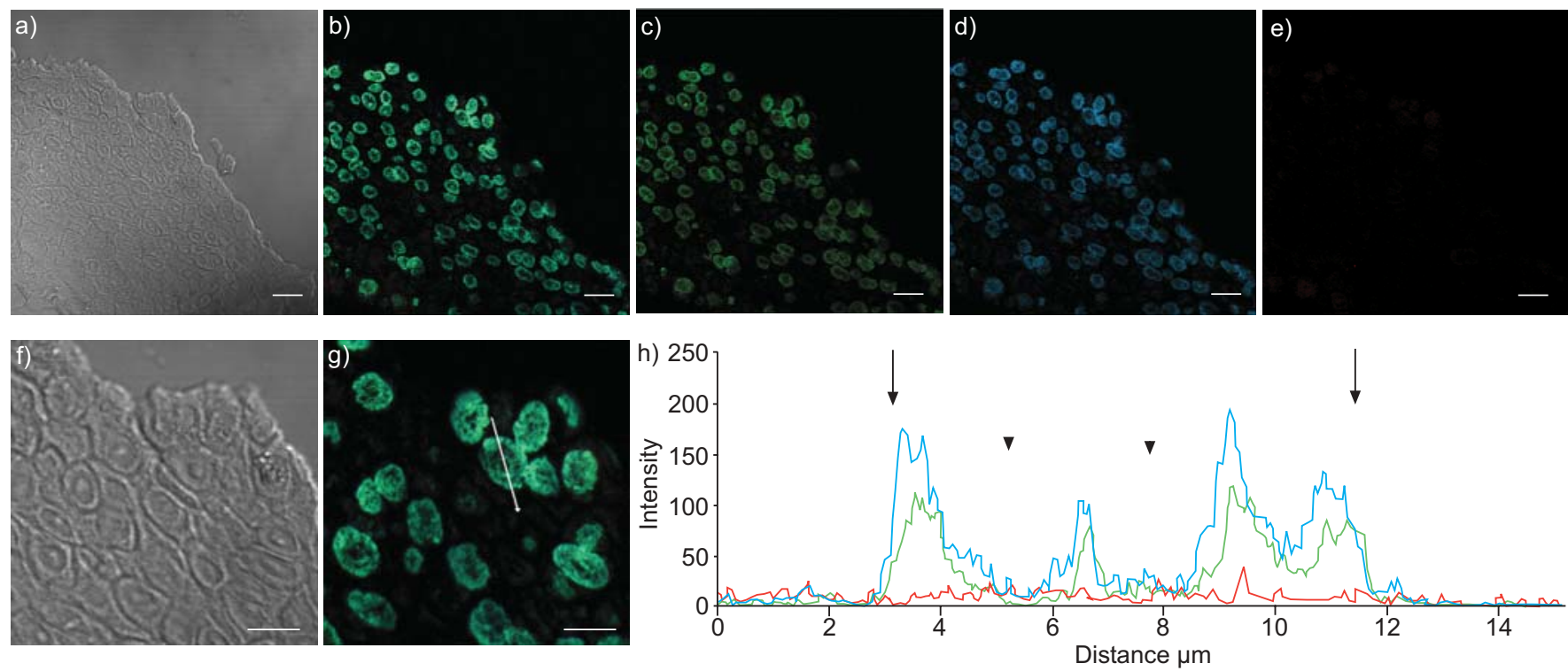

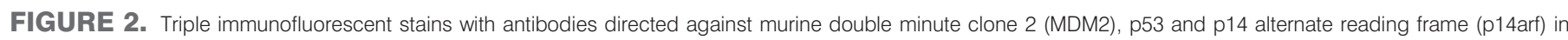

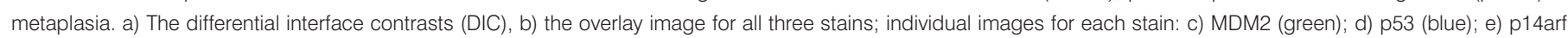

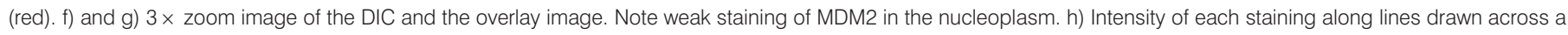

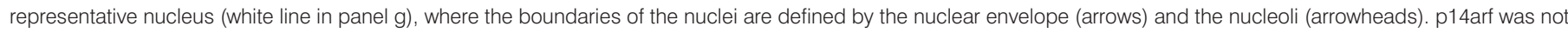

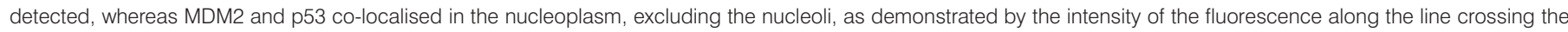
nucleus. Scale bars $=10 \mu \mathrm{m}$. 

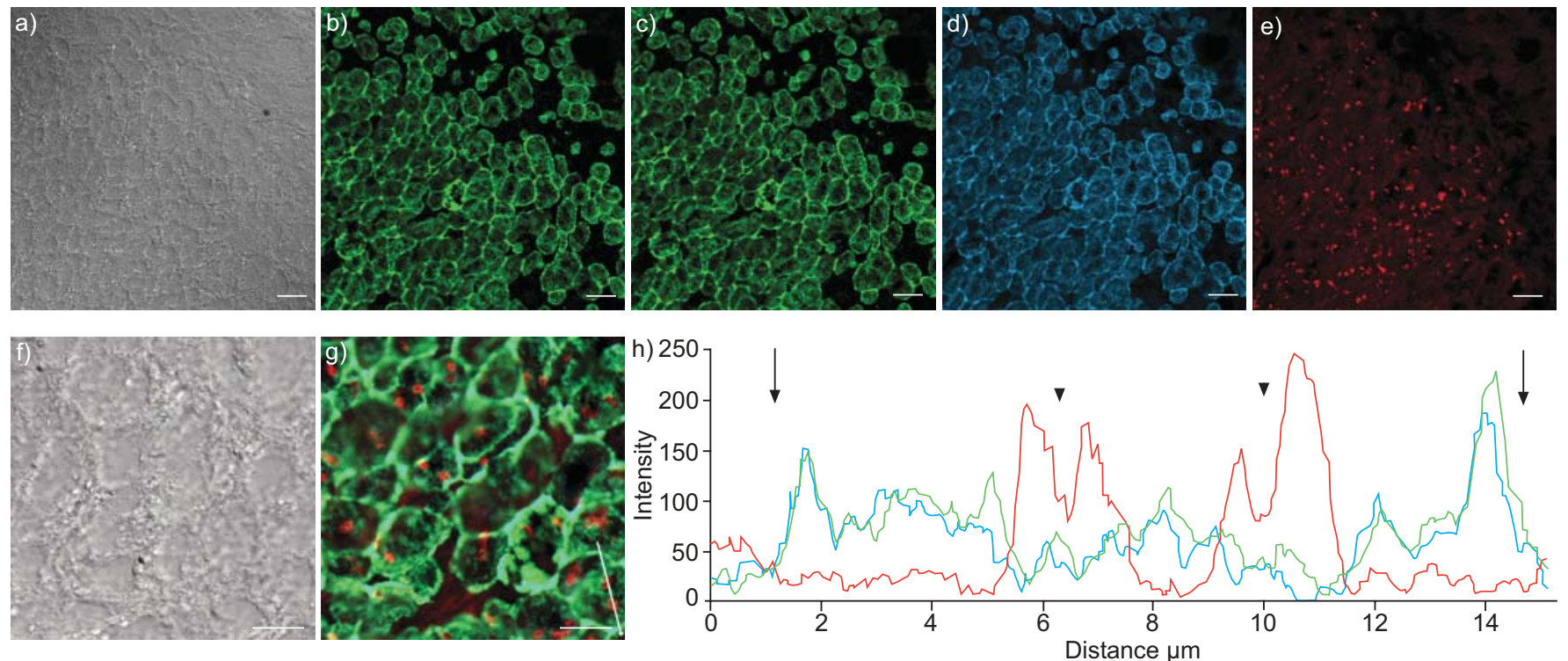

FIGURE 3. Triple immunofluorescent stains with antibodies directed against murine double minute clone 2 (MDM2), p53 and p14 alternate reading frame ( $\mathrm{p} 14$ arf) in in situ carcinoma. a) Differential interface contrasts (DIC), b) the overlay image for all three stains; individual images for each stain: c) MDM2 (green); d) p53 (blue); and e) p14arf (red). f) and g) $3 \times$ zoom image of the DIC and the overlay image. h) Intensity of each staining along lines drawn across a representative nucleus (white line in panel g), where the boundaries of the nuclei are defined by the nuclear envelope (arrows) and the nucleoli (arrowheads). MDM2 and p53 co-localised in the nucleoplasm, and p14arf was concentrated in nucleoli. Scale bars $=10 \mu \mathrm{m}$.

The current study is the first to assess MDM2 protein overexpression at every stage of SQCC development. Previously, MDM2 expression has been detected in some cases of hyperplasia, metaplasia and dysplasia, but the proportion of lesions expressing MDM2 was not determined nor compared with normal tissue expression [14]. MDM2 was initially overexpressed at the MiD stage in the current study, with $80 \%$ of the biopsies showing a high MDM2 expression, compared with only $19 \%$ of normal bronchial tissue.
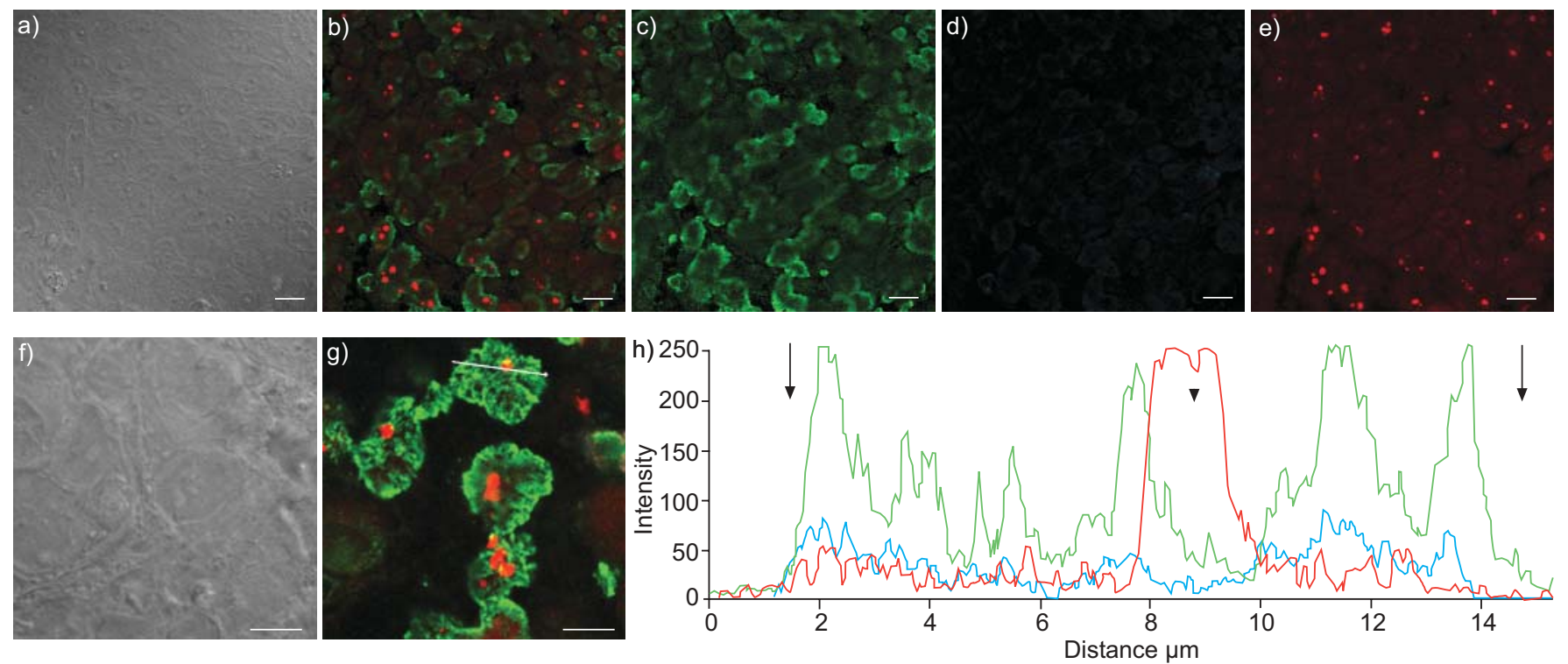

FIGURE 4. Triple immunofluorescent stains with antibodies directed against murine double minute clone 2 (MDM2), p53 and p14 alternate reading frame (p14arf) in invasive squamous cell carcinoma. a) Differential interface contrasts (DIC), b) the overlay image for all three stains; individual images for each stain: c) MDM2 (green); d) p53 (blue); e) p14arf (red). f) and g) $3 \times$ zoom image of the DIC and the overlay image. Note the more intense staining of MDM2 in the nucleoplasm in comparison to figure 2 . h) Intensity of each staining along lines drawn across a representative nucleus (white line in panel g), where the boundaries of the nuclei are defined by the nuclear envelope (arrows) and the nucleoli (arrowheads). p53 was not detected and the distribution of MDM2 and p14arf expression were similar to those illustrated in figure 3. Scale bars $=10 \mu \mathrm{m}$ 

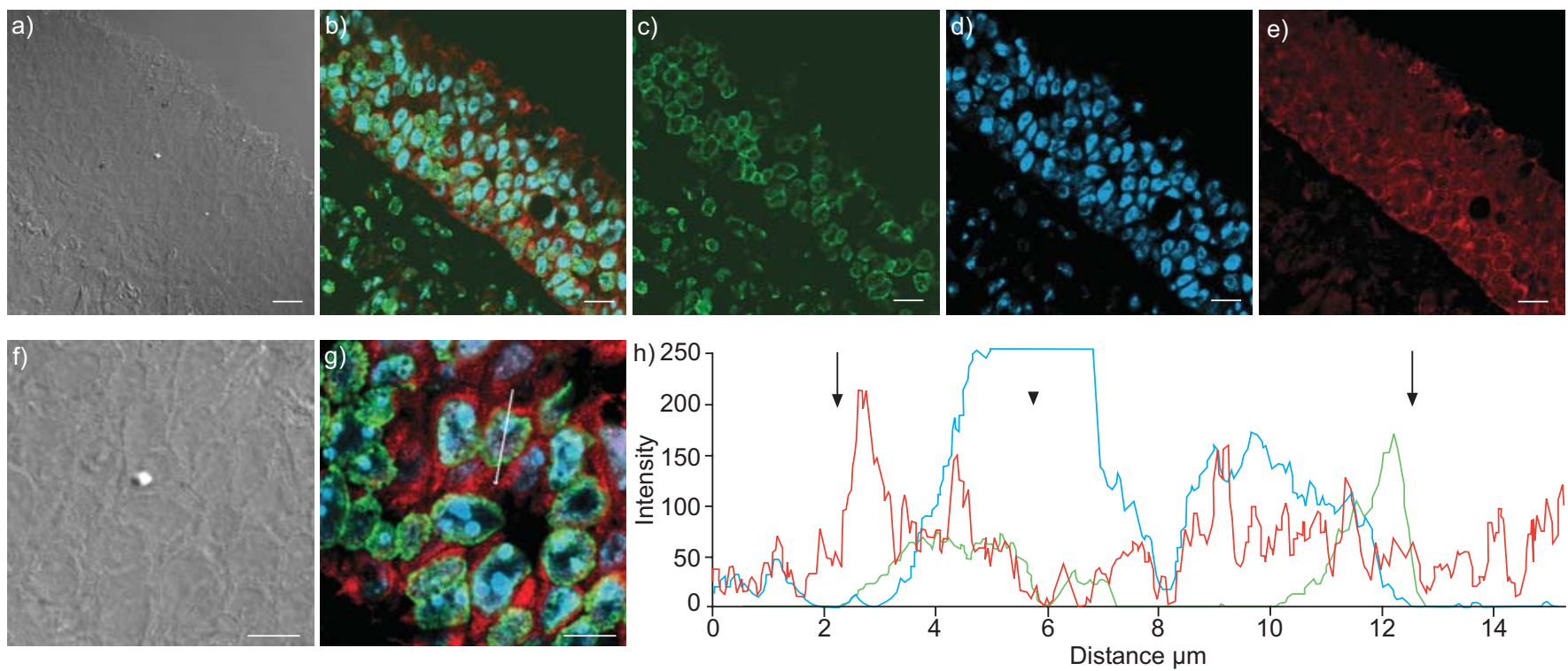

FIGURE 5. Triple immunofluorescence for murine double minute clone 2 (MDM2), nucleophosmin (NPM) and p14 alternate reading frame (p14arf) in hyperplasia a) Differential interface contrasts (DIC), b) the overlay image for all three stains; individual images for each stain: c) MDM2 (green); d) NPM (blue); e) p14arf (red). f) and g) $3 \times$ zoom image of the DIC and the overlay image. Note the more intense staining of MDM2 in the nucleoplasm in comparison to figure 2. h) Intensity of each staining along lines drawn across a representative nucleus (white line in panel g), where the boundaries of the nuclei are defined by the nuclear envelope (arrows) and the nucleoli (arrowheads). The hyperplasia reported displayed NPM detection both in the nucleoli and the nucleoplasm and p14arf co-localised with NPM in the nucleoplasm but not in the nucleoli. Scale bars $=10 \mu \mathrm{m}$

Previous studies have demonstrated a loss of p14arf expression in $18-50 \%$ of invasive SQCC $[20,22,24]$. However, no study has been published on $\mathrm{p} 14$ arf expression in bronchial squamous cell pre-neoplastic lesions. The current data revealed that p14arf loss of expression occurred at early stages. This abnormality was observed at a higher grade (SD) than MDM2 overexpression, with half of SD cases losing p14arf expression. In addition, some pre-neoplastic samples displayed a redistribution of p14arf in the nucleoli. The present study revealed that this pattern of expression was not present in normal tissue from smokers but
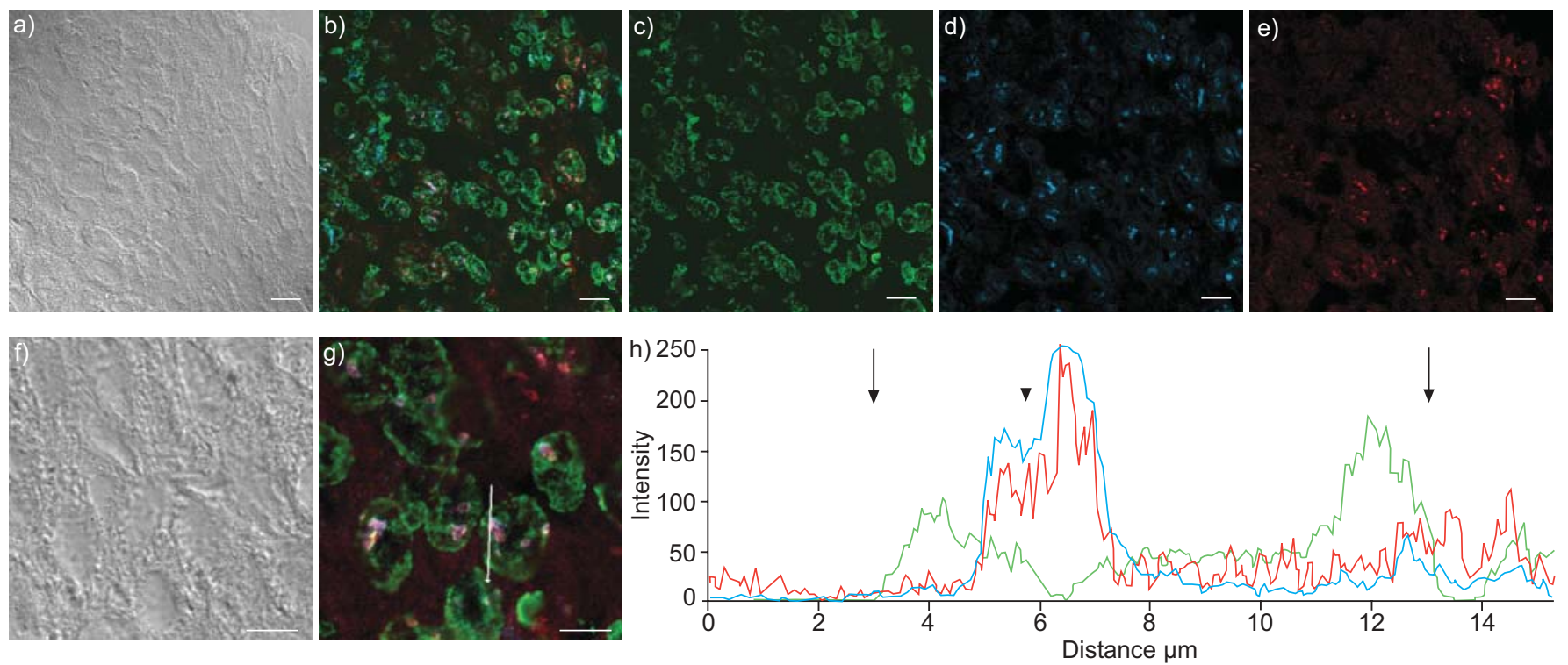

FIGURE 6. Triple immunofluorescence for murine double minute clone 2 (MDM2), nucleophosmin (NPM) and p14 alternate reading frame (p14arf) in invasive squamous cell carcinoma. a) Differential interface contrasts (DIC), b) the overlay image for all three stains; individual images for each stain: c) MDM2 (green); d) NPM (blue); e) p14arf (red). f) and g) $3 \times$ zoom image of the DIC and the overlay image. h) Intensity of each staining along lines drawn across a representative nucleus (white line in panel g), where the boundaries of the nuclei are defined by the nuclear envelope (arrows) and the nucleoli (arrowheads). An invasive carcinoma is illustrated in which NPM and p14arf are both expressed and co-localised in the nucleoli only. MDM2 remained localised in the nucleoplasm, excluding the nucleoli. Scale bars $=10 \mu \mathrm{m}$. 
increased with the severity of the lesions, most significantly at SD. Furthermore, among 46 invasive lung carcinomas on surgically resected tumours, $36 \%$ of SQCC were positive for p14arf that was also localised in the nucleoli (data not shown). The current results are based on two complementary methods: IHC and IF. Both techniques defined three patterns of p14arf expression. First, samples negative for p14arf were consistently detected by both techniques. Secondly, IF determined that the diffuse nuclear pattern observed by IHC was a distribution in the nucleoplasm, excluding the nucleoli. Thirdly, IF clearly defined the punctate distribution of p14arf and NPM as a colocalisation in the nucleoli, as identified on DIC examination and by detection of the C23 nucleolar protein (data not shown).

The two following hypotheses were formulated to explain the role of the relocalisation of p14arf. The first hypothesis is for a protective effect of p53 activity: the concentration of p14arf in the nucleoli would sequester MDM2 in the nucleoli, thereby preventing MDM2 from inhibiting p53-induced cell cycle arrest and apoptosis [4]. The second is a pejorative effect: the nucleolar localisation of p14arf, possibly in association with NPM, would prohibit its ability to form complexes with nucleoplasmic MDM2 [6]. IF staining indicated that the nucleolar localisation of p14arf did not correlate with MDM2 nucleolar relocalisation, favouring the hypothesis that the relocalisation of p14arf in the nucleoli prevents the formation of p14arf-MDM2 complexes in the nucleoplasm. The association detected by IHC between the loss of p14arf expression and its nucleolar relocalisation in conjunction with increased MDM2 levels also support this hypothesis. The low level of MDM2 expression when p14arf was expressed in the nucleoplasm could be due to the promotion of MDM degradation by p14arf [31].

As NPM is thought to play a role in cancer $[7,8]$, particularly by inducing p14arf nucleolar relocalisation [6], its expression was assessed in the same biopsies by IHC and by IF. Relocalisation of NPM has been reported in colic carcinogenesis [30]. The present experiments provide the first data on NPM expression in bronchial carcinogenesis and add to the limited data available on NPM protein expression in cancers. The entire series of normal lung tissue from smokers showed diffuse nuclear NPM distribution and p14arf co-localised with NPM in the nucleoplasm. The number of biopsies with NPM concentrated in the nucleoli (no NPM detected in the nucleoplasm) increased progressively with the severity of the lesions, with a significant cut-off at MoD. The nucleolar relocalisations of both NPM and p14arf and the absence of NPM in the nucleoplasm were strongly associated. In both normal tissue and bronchial squamous cell pre-invasive stages, MDM2 was expressed in the nucleoplasm, excluding the nucleoli. These data support the hypothesis that NPM, by relocalising p14arf to the nucleoli, might prevent p14arf from inhibiting MDM2 in the nucleoplasm [6]. However, whether NPM has a role in lung carcinogenisis and whether such a role involves the regulation of p53 through p14arf nucleolar relocalisation requires further in vitro and in vivo investigations. The work by COLOMBO and co-workers $[32,33]$ indicates that wild-type NPM, but not a leukaemia-associated NPM mutant, controls the nucleolar localisation and the stability of p14arf. The work by KORGAONKAR et al. [6] demonstrates, in addition, that NPM antagonises p14arf function by mediating its recruitment in the nucleoli. In contrast, previous results by ColOMBo et al. [34] suggest that NPM is also able to interact directly with p53, thereby increasing its stability and its transcriptional activity. These apparently contradictory results might be reconciled if NPM exerts opposite effects on p53 activity, depending on its level of expression, as recently suggested [8]: high concentration of NPM distributed in the whole nucleus would activate p53 [34], whereas low concentration of NPM, localised in the nucleoli only, would inhibit p53 [6].

In conclusion, the present data show that changes occur in murine double minute clone 2, p14 alternate reading frame and nucleophosmin level of expression and/or cellular distribution during early steps of lung carcinogenesis and, thereby, shed further light on the chronological appearance of biological abnormalities in the process of bronchial squamous cell carcinoma development.

\section{REFERENCES}

1 Martin B, Verdebout JM, Mascaux C, et al. Expression of p53 in pre-neoplastic and early neoplastic bronchial lesions. Oncol Rep 2002; 9: 223-229.

2 Momand J, Wu HH, Dasgupta G. MDM2-master regulator of the p53 tumor suppressor protein. Gene 2000; 242: 15-29.

3 Tao W, Levine AJ. p19arf stabilizes p53 by blocking nucleocytoplasmic shuttling of MDM2. Proc Natl Acad Sci USA 1999; 96: 6937-6941.

4 Weber JD, Taylor LJ, Roussel MF, Sherr CJ, Bar-Sagi D. Nucleolar arf sequesters MDM2 and activates p53. Nat Cell Biol 1999; 1: 20-26.

5 Llanos S, Clark PA, Rowe J, Peters G. Stabilization of p53 by $\mathrm{p} 14 \mathrm{ARF}$ without relocation of MDM2 to the nucleolus. Nat Cell Biol 2001; 3: 445-452.

6 Korgaonkar C, Hagen J, Tompkins V, et al. Nucleophosmin (B23) targets arf to nucleoli and inhibits its function. Mol Cell Biol 2005; 25: 1258-1271.

7 Grisendi S, Mecucci C, Falini B, Pandolfi PP. Nucleophosmin and cancer. Nat Rev Cancer 2006; 6: 493-505.

8 Gjerset RA. DNA damage, p14arf, nucleophosmin (NPM/ B23), and cancer. J Mol Histol 2006; 37: 239-2351.

9 Dworakowska D, Jassem E, Jassem J, et al. MDM2 gene amplification: a new independent factor of adverse prognosis in non-small cell lung cancer (NSCLC). Lung Cancer 2004; 43: 285-295.

10 Aikawa H, Sato M, Fujimura S, et al. MDM2 expression is associated with progress of disease and WAF1 expression in resected lung cancer. Int J Mol Med 2000; 5: 631-633.

11 Ko JL, Cheng YW, Chang SL, Su JM, Chen CY, Lee H. MDM2 mRNA expression is a favorable prognostic factor in non-small-cell lung cancer. Int J Cancer 2000; 89: 265-270.

12 Koga T, Hashimoto S, Sugio K, et al. Heterogeneous distribution of P53 immunoreactivity in human lung adenocarcinoma correlates with MDM2 protein expression, rather than with P53 gene mutation. Int J Cancer 2001; 95: 232-239.

13 Higashiyama M, Doi O, Kodama K, et al. MDM2 gene amplification and expression in non-small-cell lung cancer: immunohistochemical expression of its protein is a 
favourable prognostic marker in patients without p53 protein accumulation. Br J Cancer 1997; 75: 1302-1308.

14 Rasidakis A, Orphanidou D, Kalomenidis J, et al. Expression of MDM2 protein in neoplastic, pre-neoplastic, and normal bronchial mucosa specimens: comparative study with p53 expression. Hybridoma 1998; 17: 339-345.

15 Mori S, Ito G, Usami N, et al. p53 apoptotic pathway molecules are frequently and simultaneously altered in nonsmall cell lung carcinoma. Cancer 2004; 100: 1673-1682.

16 Marchetti A, Buttitta F, Pellegrini S, et al. MDM2 gene amplification and overexpression in non-small cell lung carcinomas with accumulation of the p53 protein in the absence of p53 gene mutations. Diagn Mol Pathol 1995; 4: 93-97.

17 Gorgoulis VG, Zacharatos P, Kotsinas A, et al. Altered expression of the cell cycle regulatory molecules $\mathrm{pRb}, \mathrm{p} 53$ and MDM2 exert a synergetic effect on tumor growth and chromosomal instability in non-small cell lung carcinomas (NSCLCs). Mol Med 2000; 6: 208-237.

18 Gugger M, Kappeler A, Vonlanthen S, et al. Alterations of cell cycle regulators are less frequent in advanced nonsmall cell lung cancer than in resectable tumours. Lung Cancer 2001; 33: 229-239.

19 Xue Q, Sano T, Kashiwabara K, Saito M, Oyama T, Nakajima T. Aberrant expression of pRb, p16, p14ARF, MDM2, p21 and p53 in stage I adenocarcinomas of the lung. Pathol Int 2002; 52: 103-109.

20 Xue Q, Sano T, Kashiwabara K, Oyama T, Nakajima T. Aberrant expression of pRb, p16, p14arf, MDM2, p21 and p53 in squamous cell carcinomas of lung. Jpn J Cancer Res 2001; 92: 285-292.

21 Eymin B, Gazzeri S, Brambilla C, Brambilla E. MDM2 overexpression and p14arf activation are two mutually exclusive events in primary human lung tumors. Oncogene 2002; 21: 2750-2761.

22 Hsu HS, Wang YC, Tseng RC, et al. 5' cytosine-phosphoguanine island methylation is responsible for p14ARF inactivation and inversely correlates with p53 overexpression in resected non-small cell lung cancer. Clin Cancer Res 2004; 10: 4734-4741.

23 Wang YC, Lin RK, Tan YH, Chen JT, Chen CY, Wang YC. Wild-type p53 overexpression and its correlation with MDM2 and p14ARF alterations: an alternative pathway to non-small-cell lung cancer. J Clin Oncol 2005; 23: 154-164.
24 Gazzeri S, Della Valle V Chaussade L, Brambilla C, Larsen CJ, Brambilla E. The human p19arf protein encoded by the beta transcript of the p16INK4a gene is frequently lost in small cell lung cancer. Cancer Res 1998; 58: 39263931.

25 Sano $\mathrm{T}$, Hikino $\mathrm{T}$, Xue $\mathrm{Q}$, et al. Immunohistochemical inactivation of p14arf concomitant with MDM2 overexpression inversely correlates with p53 overexpression in oral squamous cell carcinoma. Pathol Int 2000; 50: 709-716.

26 Vermylen P, Pierard P, Roufosse C, et al. Detection of bronchial pre-neoplastic lesions and early lung cancer with fluorescence bronchoscopy: a study about its ambulatory feasibility under local anaesthesis. Lung Cancer 1999; 25: 161-168.

27 Travis WD, Colby TV, Shimosato Y, et al., World Health Organization/International Histological Classification of Lung and Pleural Tumors. 3rd Edn. Berlin, Spinger-Verlag, 1999.

28 King G, Payne S, Walker F, Murray GI. A highly sensitive detection method for immunohistochemistry using biotinylated tyramine. J Pathol 1997; 183: 237-241.

29 Mascaux C, Martin B, Verdebout JM, Ninane V, Sculier JP. COX-2 expression during early lung squamous cell carcinoma oncogenesis. Eur Respir J 2005; 26: 198-203.

30 Nozawa Y, Van BN, Van der Made AC, Dinjens WN, Bosman FT. Expression of nucleophosmin/B23 in normal and neoplastic colorectal mucosa. J Pathol 1996; 178: 48-52.

31 Zhang Y, Xiong Y, Yarbrough WG. ARF promotes MDM2 degradation and stabilizes p53: ARF-INK4a locus deletion impairs both the $\mathrm{Rb}$ and p53 tumor suppression pathways 7. Cell 1998; 92: 725-734.

32 Colombo E, Martinelli P, Zamponi R, et al. Delocalization and destabilization of the arf tumor suppressor by the leukemia-associated NPM mutant 5. Cancer Res 2006; 66: 3044-3050.

33 Colombo E, Bonetti P, Lazzerini DE, et al. Nucleophosmin is required for DNA integrity and p19arf protein stability. Mol Cell Biol 2005; 25: 8874-8886.

34 Colombo E, Marine JC, Danovi D, Falini B, Pelicci PG. Nucleophosmin regulates the stability and transcriptional activity of p53. Nat Cell Biol 2002; 4: 529-533. 